\title{
A Graph Clustering Algorithm Based on the Intra Vertex Adjacent Ratio
}

\author{
Haili Guo ${ }^{1}$ and Deqiang Wang ${ }^{2 *}$ \\ ${ }^{1}$ Beijing City University, Beijing, P.R. China \\ ${ }^{2}$ Dalian Maritime University, Dalian, P.R. China \\ ${ }^{*}$ Corresponding author
}

\begin{abstract}
This paper proposes a new clustering coefficient based on the number of connecting vertices within a cluster (called inter vertex adjacent ratio, IVAR) and proposes a new clustering algorithm based on IVAR. Finally, the analysis of algorithm applicability shows that the algorithm is more applicative and effective for regular graphs than the vertex-clustering algorithm based on intra connection ratio [L. Moussiades and A. Vakali, Clustering dense graph: A web site graph paradigm. Information Processing and Management, vol. 46, pp.247-267, 2010].
\end{abstract}

Keywords-component; graph; clustering algorithm; intra vertex connection ratio

\section{INTRODUCTION}

Clustering is an important tool for data analysis and processing, has been widely used in many fields. Graphclustering is an important variant of data clustering. According to some given index, by Graph-clustering, the graph can be divided into several subgraphs which contain relatively closely connected vertices and their associated edge.

Graph-clustering algorithm plays an important role in many fields: monitoring on computer network executive purpose, visualization knowledge based on support of understanding of complex data structure, measurement data cluster, detection source code plagiarism, network data cluster and online community identification ${ }^{[3,5,6,7]}$. Clustering based on graph theory is one of the clustering method developed rapidly recently, which is based on graph theory and computer graphics ${ }^{[1,2,4,8]}$. Graph-clustering is a very widely applied research topic, especially in the network research, including email network, social network, gene networks and so on.

Generally, graph-clustering clusters are groups with a high density of within-group edges and a lower density of betweengroup edges. Moussiades and Vakali proposed a vertexclustering algorithm based on intra connection ratio (we call the algorithm as MV-ICR algorithm) in Ref. [7]. A graph can be clustered according to the degree of internal vertex by MVICR. For the internal vertices of a class, classification index is not only related with the degree of a vertex, but also the number of the external vertices connected to the class. This paper proposes a new clustering algorithm based on the ratio of the number of connecting vertices, and the algorithm is more reasonable and effective than MV-ICR algorithm for some especial graphs, such as regular graph.

\section{DEFINITIONS AND NotATIONS}

Let $G=(V, E)$ be a graph, a clustering of $G$ vertices is denoted as $\mathscr{C}(G)$, a clustering of $G$ vertices, the element of $\mathscr{C}(G)$ is called a cluster. $\forall v \in V(G)$, the degree of $v$ is denoted as $d(v)$. The following definitions and notations are mainly from Ref. [7].

Definition 1. Let $C \in \mathscr{C}(G)$ be a clustering of $G$, for any $C \in \mathscr{C}(G),|C| /(|C|+|N(C)|)$ is called the inter vertex adjacent ratio, denoted as $\operatorname{ivar}(C)$, where

$$
N(C)=\{v \mid(u, v) \in E(G), u \in C, v \notin C\}
$$

Definition 2. Let $\mathscr{C}(G)$ be a clustering of $\mathbf{G}$, for any $\mathbf{C}$, $\mathbf{S} \in \mathscr{C}(G)$,

$$
\frac{|C U S|}{|C U S|+\left|N_{G}(C U S)\right|}-\frac{|C|}{|C|+\left|N_{G}(C)\right|}-\frac{|S|}{|S|+\left|N_{G}(S)\right|}
$$

is called inter vertex adjacent ratio of merging $C$ and $S$ ( C US ), denoted as $\Delta_{I V A R}(C, S)\left(\Delta_{I V A R}\right.$ for short).

For $C=\{u\}, S=\{v\}$, the inter vertex adjacent ratio of $C \mathrm{US}$ is

$$
\Delta_{I V A R}(u, v)=\frac{2}{2+|N(\{u, v\})|}-\frac{1}{1+d(u)}-\frac{1}{1+d(v)}
$$

\section{Clustering AlgORITHM BASED ON IVAR}

A. A Graph-Clustering Algorithm Based on IVAR IVAR

Algorithm 1: A graph-clustering algorithm based on

S1: INPUT graph $G=(V, E)$

S2: Let $\mathscr{C}(G)=\left\{C_{1}, C_{2}, \ldots, C_{|V|}\right\}, C_{i}=\left\{v_{i}\right\}$

S3: $\forall C_{i}, C_{j} \in \mathscr{C}(G)$, if $d\left(C_{i}, C_{j}\right)>0$, then 


$$
\begin{aligned}
\Delta_{\text {IVAR }}\left(C_{i}, C_{j}\right) & =\frac{\left|C_{i} \mathrm{U} C_{j}\right|}{\left|C_{i} \mathrm{U} C_{j}\right|+\left|N\left(C_{i} \mathrm{U} C_{j}\right)\right|} \\
& -\frac{\left|C_{i}\right|}{\left|C_{i}\right|+\left|N\left(C_{i}\right)\right|}-\frac{\left|C_{j}\right|}{\left|C_{j}\right|+\left|N\left(C_{j}\right)\right|} ;
\end{aligned}
$$

\section{S4: IF $|\mathscr{C}(G)|>1$ THEN}

a. finding the cluster subset with the maximum $\Delta_{\text {IVAR }}$.

b. if it does not exist, then exit.

c. if there is a cluster subset $\left\{C_{i_{1}}, C_{i_{2}}, \ldots, C_{i_{k}}\right\}(k \geq 3)$ with the maximum $\Delta_{\text {IVAR }}$ and equal $\Delta_{\text {IVAR }}$ among each other, then merge those clusters into $S$ :

$$
\begin{gathered}
S=\mathrm{U}_{j=1}^{k} C_{i_{j}}, \\
\mathscr{C}(G)=\left(\mathscr{C}(G)-\left\{C_{i_{1}}, C_{i_{2}}, \mathrm{~L}, C_{i_{k}}\right\}\right) \mathrm{U} S ;
\end{gathered}
$$

d. else merge $C_{i}, C_{j}$ with the maximum $\Delta_{\text {IVAR }}$ into $S$ :

$$
S=C_{i} \mathrm{U} C_{j}, \mathscr{C}(G)=\left(\mathscr{C}(G)-\left\{C_{i}, C_{j}\right\}\right) \mathrm{U} S_{;}
$$

ع. $\forall C_{i}, C_{j} \in \mathscr{C}(G), \imath \phi^{d\left(C_{i}, C_{j}\right)>0}, \chi \alpha \lambda \chi \nu \lambda \alpha \tau \varepsilon \Delta_{I V A R}\left(C_{i}, C_{j}\right)$.

\section{S5: END}

\section{B. Example Based on IVAR}

We cluster the vertices of the graph $G$ in Figure I with Algorithm 1 as follows:

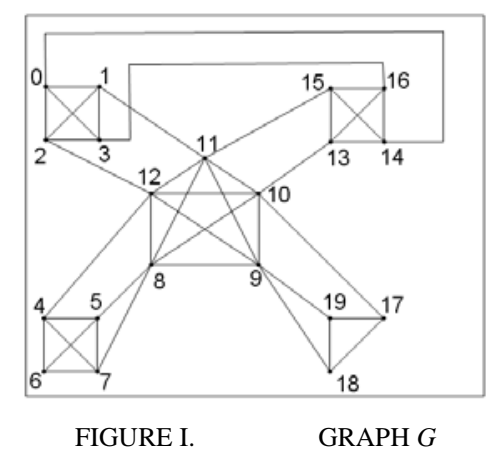

(1) Every vertex in the graph is a cluster $\mathscr{C}=\{\{0\},\{1\},\{2\}, \ldots,\{19\}\}$, then calculate $\Delta_{\text {IVAR }}$. Because $\Delta_{\text {IVAR }}(5,7)=\Delta_{\text {IVAR }}(18,19)$ is the maximum value, we choose one pair of $\{18\}$ and $\{19\}$, merging into one cluster $\{18,19\}$ (see Figure II) and get the clustering as follows:

$$
\begin{aligned}
& S=\{18\} U\{19\}=\{18,19\} \\
& \mathscr{C}=(\mathscr{C}-\{\{18\},\{19\}\}) \cup S=\{\{1\},\{2\}, \ldots,\{17\},\{18,19\}\} .
\end{aligned}
$$

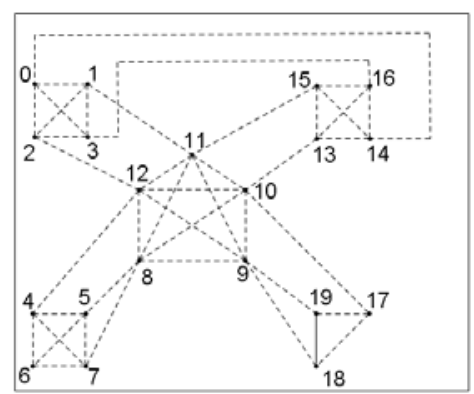

FIGURE II. MERGING $\{18\}$ AND (19) TOGETHER

(2) Calculating $\Delta_{\text {IVAR }}$ of $\mathscr{C}$, and finding the maximum value $\Delta_{\text {IVAR }}(5,7)$, then we merge $\{5\}$ and $\{7\}$ (see Figure III), get the clustering as follows:

$$
\mathscr{C}=\{\{0\},\{1\},\{2\},\{3\},\{4\},\{5,7\},\{6\},\{8\},\{9\}, \ldots,\{17\},\{18,19\}\} .
$$

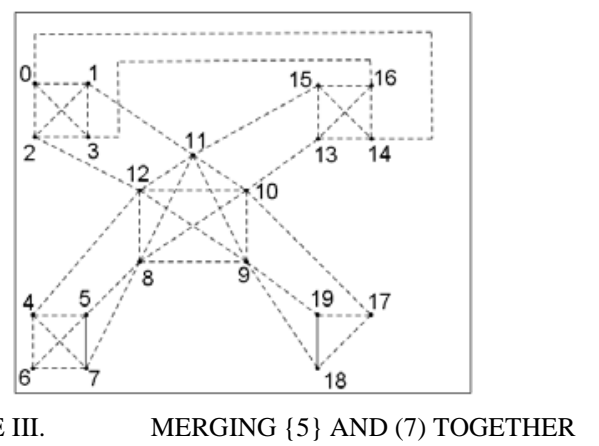

FIGURE III.

(3) Calculating $\Delta_{\text {IVAR }}$ of $\mathscr{C}$, and finding the maximum value $\Delta_{I V A R}(\{5,7\},\{6\})$, then we merge $\{5,7\}$ and $\{6\}$ (see Figure IV), and get the clustering as follows:

$$
\mathscr{C}=\{\{0\}\{1\},\{2\},\{3\},\{4\},\{5,6,7\},\{8\},\{9\}, \ldots,\{17\},\{18,19\}\}
$$

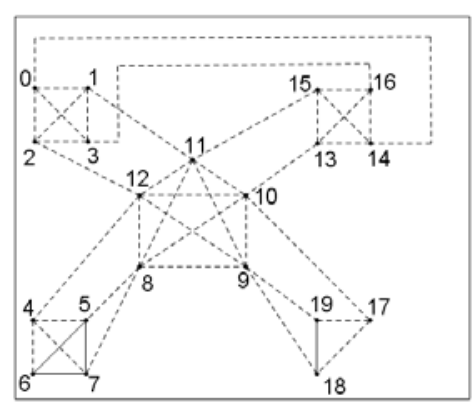

FIGURE IV. MERGING $\{5,7\}$ AND $\{6\}$ TOGETHER 
(4) Calculating $\Delta_{I V A R}$ of $\mathscr{C}$, the $\Delta_{\text {IVAR }}$ between each pair of $\{8\},\{9\},\{10\},\{11\}$ and $\{12\}$ is maximum, then we merge them into one cluster of $\{8,9,10,11,12\}$ (see Figure V), and get the cluster as follows:

$$
\begin{array}{r}
\mathscr{C}=\{\{0\},\{1\},\{2\},\{3\},\{4\},\{5,6,7\},\{8,9,10,11,12\}, \\
\{13\},\{14\}, \ldots,\{17\},\{18,19\}\} .
\end{array}
$$

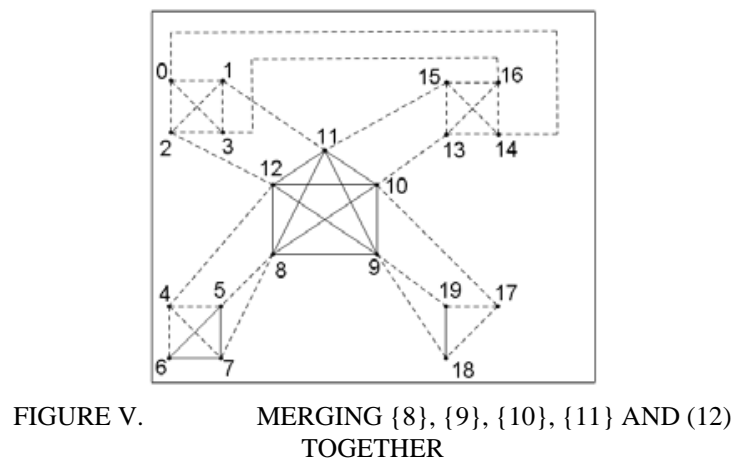

(5) Calculating $\Delta_{I V A R}$ of $\mathscr{C}$, the $\Delta_{\text {IVAR }}$ between each pair of $\{0\},\{1\},\{2\},\{3\}$ is maximum, then we merge them into one cluster of $\{0,1,2,3\}$ (see Figure VI), and get the cluster as follows:

$$
\begin{array}{r}
\mathscr{C}=\{\{0,1,2,3\},\{4\},\{5,6,7\},\{8,9,10,11,12\}, \\
\{13\},\{14\}, \ldots,\{17\},\{18,19\}\} .
\end{array}
$$

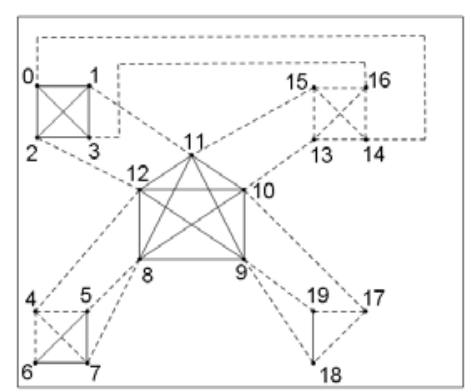

FIGURE VI. MERGING $\{0\},\{1\},\{2\}$ AND (3) TOGETHER

(6) Calculating $\Delta_{I V A R}$ of $\mathscr{C}$, the $\Delta_{I V A R}$ between each pair of $\{13\},\{14\},\{15\},\{16\}$ is maximum, then we merge them into one cluster of $\{13,14,15,16\}$ (see Figure VII), and get the cluster as follows:

$$
\begin{array}{r}
\mathscr{C}=\{\{0,1,2,3\},\{4\},\{5,6,7\},\{8,9,10,11,12\}, \\
\{13,14,15,16\},\{17\},\{18,19\}\} .
\end{array}
$$

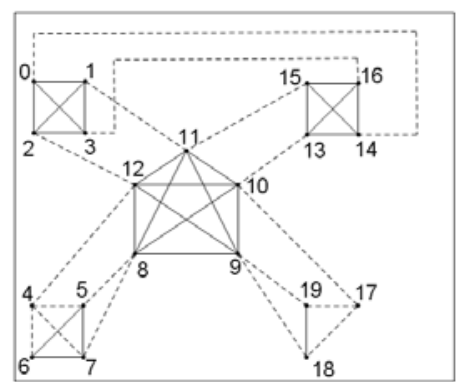

FIGURE VII.

MERGING $\{13\},\{14\},\{15\}$ AND $\{16\}$ TOGETHER

(7) Calculating $\Delta_{I V A R}$ of $\mathscr{C}$, the $\Delta_{I V A R}$ between $\{4\}$ and $\{5$, $6,7\}$ is maximum, then we merge them into one clustering of $\{4,5,6,7\}$, and get the cluster as follows:

$$
\begin{aligned}
\mathscr{C}=\{\{0,1,2,3\},\{4,5,6,7\},\{8,9,10,11,12\}, \\
\{13,14,15,16\},\{17\},\{18,19\}\} .
\end{aligned}
$$

(8) Calculating $\Delta_{I V A R}$ of $\mathscr{C}$, the $\Delta_{\text {IVAR }}$ between $\{17\}$ and $\{18,19\}$ is maximum, then we merge them into one cluster of $\{17,18,19\}$, and get the cluster as follows:

$$
\begin{array}{r}
\mathscr{C}=\{\{0,1,2,3\},\{4,5,6,7\},\{8,9,10,11,12\}, \\
\{13,14,15,16\},\{17,18,19\}\} .
\end{array}
$$

(9) Calculating $\Delta_{\text {IVAR }}$ of $\mathscr{C}$, the $\Delta_{\text {IVAR }}$ between $\{0,1,2,3\}$ and $\{8,9,10,11,12\}$ is maximum, then we merge them into one cluster of $\{0,1,2,3,8,9,10,11,12\}$, and get the cluster as follows:

$$
\begin{array}{r}
\mathscr{C}=\{\{0,1,2,3,8,9,10,11,12\},\{4,5,6,7\}, \\
\{13,14,15,16\},\{17,18,19\}\} .
\end{array}
$$

(10) Calculating $\Delta_{I V A R}$ of $\mathscr{C}$, the $\Delta_{I V A R}$ between $\{0,1,2,3$, $8,9,10,11,12\}$ and $\{4,5,6,7\}$ is maximum, then we merge them into one cluster and get the cluster as follows:

$$
\begin{array}{r}
\mathscr{C}=\{\{0,1,2,3,4,5,6,7,8,9,10,11,12\}, \\
\{13,14,15,16\},\{17,18,19\}\} .
\end{array}
$$

(11) Calculating $\Delta_{\text {IVAR }}$ of $\mathscr{C}$, the $\Delta_{\text {IVAR }}$ between $\{0,1,2,3$, $4,5,6,7,8,9,10,11,12\}$ and $\{13,14,15,16\}$ is maximum, then we merge them into one cluster and get the cluster as follows:

$$
\begin{array}{r}
\mathscr{C}=\{\{0,1,2,3,4,5,6,7,8,9,10,11,12,13,14,15,16\}, \\
\{17,18,19\}\} .
\end{array}
$$


(12) Finally, all vertices will be merged into one cluster:

$$
\mathscr{C}=\{\{0,1,2,3,4,5,6,7,8,9,10,11,12,13,14,15,16,17,18,19\}\} .
$$

\section{ANALYSIS OF ALGORITHM APPLICABILITY}

(1) IVAR is better than MV-ICR for those graphs containing the complete subgraph.

We can cluster the $K_{5}$ according to MV-ICR (Ref.[7]), and get the clustering approach shown in Figure VIII.

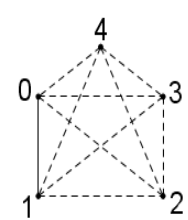

FIGURE VIII.
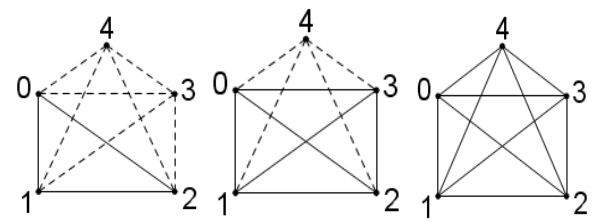

CLUSTERING PROCESS OF K5 WITH MV-ICR.
We can cluster the $K_{5}$ according to IVAR: the initial clustering is $\{0\},\{1\},\{2\},\{3\},\{4\}$; Calculating $\Delta_{\text {IVAR }}$ between all clusters, they are equal between each pair, then $\{0\},\{1\}$, $\{2\},\{3\},\{4\}$ will be merged into one cluster, and all vertices will be merged into one cluster. This algorithm can not only improve the clustering efficiency, but also make clustering more reasonable.

(2) IVAR is better than MV-ICR for regular graph.

IVAR is an algorithm based on the number of vertices, while MV-ICR is an algorithm based on the degree of a vertex. For some especial graphs, such as regular graph, an algorithm based on the degree of a vertex is inapplicable. The graph in Figure IX(g) is a 3 regular graph, that is, the degree of all vertices in graph is $d(v)=3$. Clustering according to MV-ICR, the $\Delta_{\text {IVAR }}$ is equal between each pair of vertices $\left(\Delta_{\text {ICR }}\left(\left\{v_{i}\right\},\left\{v_{j}\right\}\right)=\frac{2}{d\left(v_{i}\right)+d\left(v_{j}\right)}\right)$, that make the choice more difficult.

If clustering the graph vertices according to IVAR, we can get the clustering process illustrated in Figure IX.

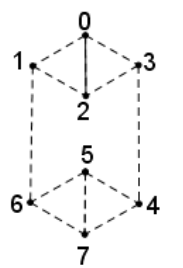

(a)

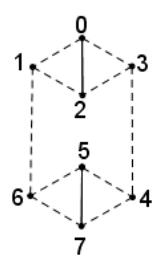

(b)

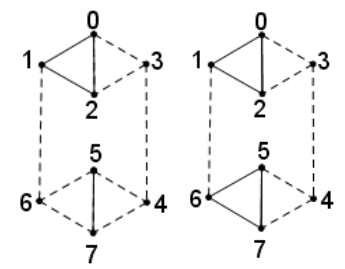

(c)

(d)

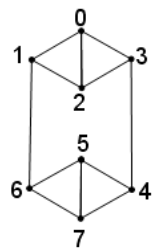

(e)<smiles>O=C1C2C3C1C1C2C31</smiles>

(f)<smiles>O=C1C2C3C1C1C2C31</smiles>

(g)
FIGURE IX.

CLUSTERING PROCESS

\section{CONCLUSION}

In the clustering coefficient field, this paper studies merely on graph-clustering related to degree of vertex and the number of vertex, without considering the other parameters.We will discuss the clustering algorithm based on or combined with other parameters in the future research. This paper only discusses the applicability of MV-ICR and IVAR clustering algorithm for simple graphs, we will probe into the applicability for general graphs to find a new graph-clustering algorithm with wild adaptability.

\section{ACKNOWLEDGMENT}

This work was financially supported by Beijing Higher Education Young Elite Teacher Project (YETP1851), and Research Project Funded by the Department of Education of Liaoning Province (L2015063).

\section{REFERENCES}

[1] P. Eades, M.L. Huang, "Navigating clustered graphs using forcedirected methods," Journal of Graph Algorithms and Applications, vol. 4(3), pp.157-181, 2002.

[2] S. Günter, H.Bunke, "Self-organizing map for clustering in the graph domain,” Pattern Recognition Letters, vol. 23, pp.401-417, 2002.

[3] V.G. Kaburlasos, L. Moussiades, A. Vakali, "Granular graph clustering in the web," in Proceedings of the 8th international conference on natural computing, Utah: World Scientific Publishing, 2007, 1639-1645.

[4] B. Luo, R.C. Wilson, E.R. Hancock, "Spectral embedding of graphs," Pattern Recognition, vol.36(10), pp. 2213-2223, 2003.

[5] L. Moussiades, A. Vakali, "Clustering dense graph: A web site graph paradigm," Information Processing and Management, vol. 46, pp.247267, 2010.

[6] L. Moussiades, A.Vakali, "PDetect: A clustering approach for detecting plagiarism in source code datasets," The Computer Journal, vol. 48(6), pp. 651-661, 2005.

[7] G. Palla, I. Dernyi, I. Farkas, "Uncovering the overlapping community structure of complex networks in nature and society," Nature, vol. 435, pp.814-818, 2005

[8] F. Serratosa, R.Alquezar, A.Sanfeliu, "Synthesis of function-described graphs and clustering of attributed graphs,” International Journal of Pattern Recognition and Artificial Intelligence, vol.16, pp. 621-655, 2002. 